\title{
Evaluation of three methods for supply chain modelling
}

\author{
J.H. Trienekens ${ }^{1)}$, H.H. Hvolby ${ }^{2)}$ \\ ${ }^{1)}$ Department of management Studies, \\ University of Wageningen, Hollandseweg 1, $6706 \mathrm{KN}$, \\ Wageningen, The Netherlands \\ e-mail:jack.trienekens@alg.bk.wau.nl \\ ${ }^{2)}$ Associate Professor, Department of Production, \\ University of Aalborg, Fibigerstraede 16, 9220 Aalborg East, \\ Denmark
}

e-mail:i9hhh@iprod.auc.dk

\begin{abstract}
In this paper three different approaches to Supply Chain Modelling are presented, the Event Process Chain (EPC), the Activity Chain Model (ACM), and the GRAI Grid method. The three methods will be described, after which application of the methods to a case will be presented. The paper will conclude with a comparison of the methods and a discussion of their complementarity.
\end{abstract}

Keywords

Supply chain, process modelling, redesign

\section{INTRODUCTION: MODELLING OF SUPPLY CHAINS}

The idea to consider business processes as a chain is not new. In 1985 Porter introduced his "Generic Value Chain" to support analysis of value added processes in companies. Since the end of the 1980s many studies followed concerning management of supply chains in which more than a single company is involved. These studies included objectives like: lead time reduction (e.g. Lee et al., 1998), cost reduction (e.g. Lalonde \& Pohlen, 1996), improvement of product quality and attention to environmental aspects (Ziggers \& Trienekens, 1998).

Also much interest has arisen in integrating various processes in the chain: decision structures and co-ordination mechanism are important issues in this regard. In this 
paper three modelling methods, covering major aspects of goods flow management will be presented:

- Event Process Chain modelling (EPC, Kim 1995) aiming at lead time reduction.

- GRAI modelling (Doumeingts., 1985) aiming at improvement of decision structures in supply chains.

- Activity Chain Modelling (ACM, Hvolby \& Barfod, 1998) aiming at optimisation of use of resources (cost reduction).

\section{DESCRIPTION OF MODELLING METHODS}

\subsection{Case supply chain}

The case supply chain includes a retailer (distribution centre and outlets) and food industries. Goal of the case analysis is to reduce lead time reduction, to gear various decision processes and to improve efficient use of resources. In the case 3 retailers and 4 food industries in the Netherlands are involved. Information needed was gathered by interviewing management employees in various logistics and information systems functions in the organisations involved. The description below is of one typical chain representable for all the retail and food industry companies involved.

\subsection{EPC Modelling}

The goal of EPC modelling is throughput time reduction in process chains with the aim to improve delivery performance, lower stock levels and increase efficiency of processes in the chain. EPC modelling focuses on processes that are directly linked to the chain output demanded by the customer. This requested output should be the starting point of chain analysis: the information flow should be traced upstream the chain and subsequently the flow of products downstream the chain. Essential in this approach is that no difference is made between physical and administrative processes. For example an order process may play an equal part in a goods flow analysis as a distribution process. The chain model used is constructed around the dimensions of place and time. Kim (1995) defines the following principles for redesign: reduction of the number of processes, transformation of processes into events, minimisation of travel distances, parallel processes/events, reduction of wait time before processes but elimination of wait time before events. In (re)design of processes with EPC modelling information and communication technology plays a significant factor. 


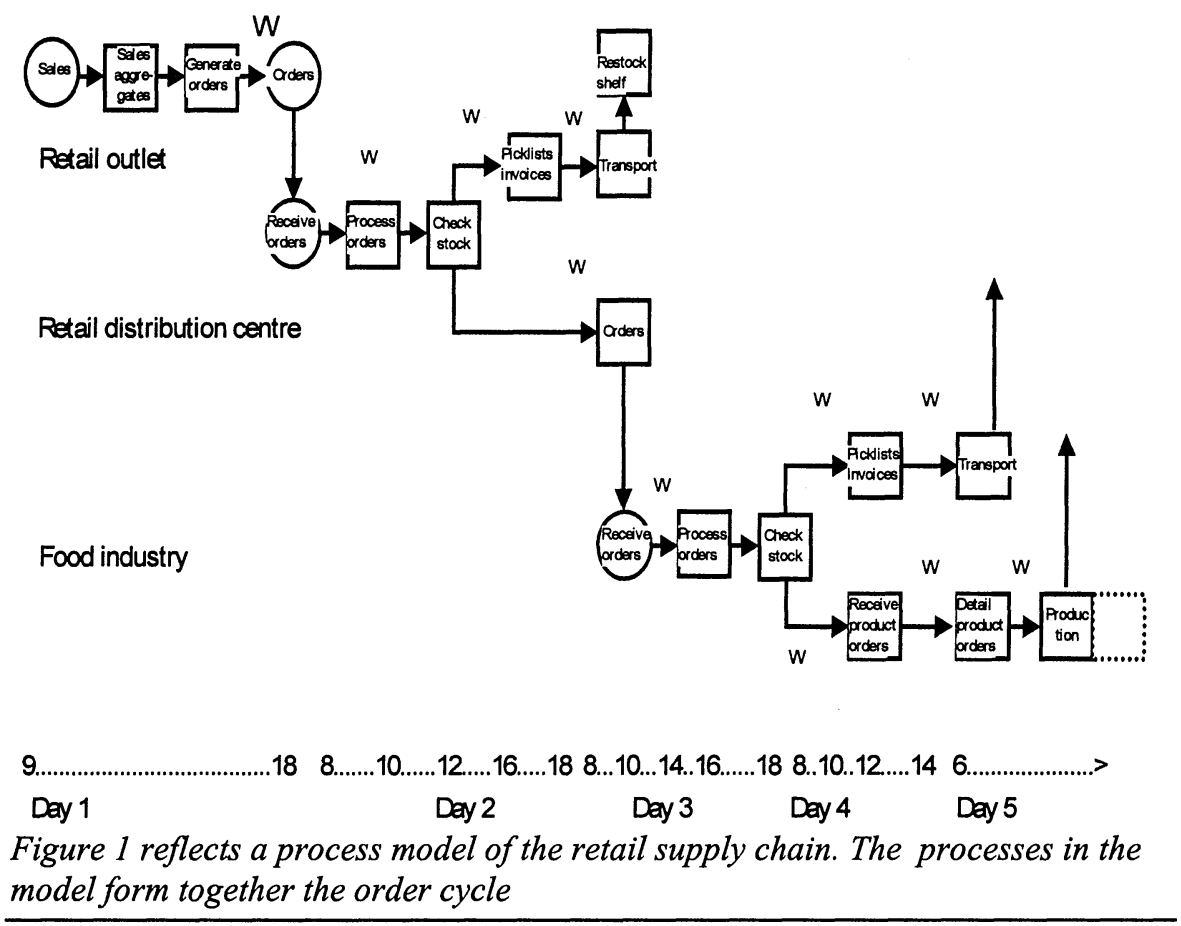

[In the figure a circle is an event with no time consumption, a square is a process with time consumption, a ' $w$ ' reflects waiting time]

Major improvement options in the case:

- reduction of the number of processes: making up orders at the retail store manually can be replaced by automated ordering; ordering at the distribution centre can be skipped if the food industry is responsible for inventory control.

- transformation of processes in events and shortening of processes: automatization of various processes (e.g inventory checks) and decision support systems for production planning.

- parallellization of processes: scanned sales data of the retail outlets can be sent directly to the other chain parties, meaning that processes can already start before actual orders come in.

- reduction of waiting times: real time processing of orders, more frequent deliveries throughout the supply chain.

Results of a pilot project showed throughput time reduction from six to two days, increase of the total product freshness of five days, reduction of inventory at the distribution centre of $55 \%$ and at the retail outlet of $38 \%$ (Vorst et al., 1998). 


\subsection{GRAI Grids}

Through a GRAI Grid (Doumeingts, 1985; Coll et al., 1998) relationships between decisions, decision flows and the required information flows can be identified. The GRAI Grid is designed to describe the decision making processes within a company. In the grid every cell can be regarded as a potential decision centre (nodes that receive inputs in the form of decision frames and information, and make resultant frames and information). A decision centre can be period driven or event driven. The grid is hierarchical, ranging from strategic decisions with long intervals, to frequently made decisions in real time. Each decision centre has one or more objectives that are to be achieved by a certain time- the 'horizon'.

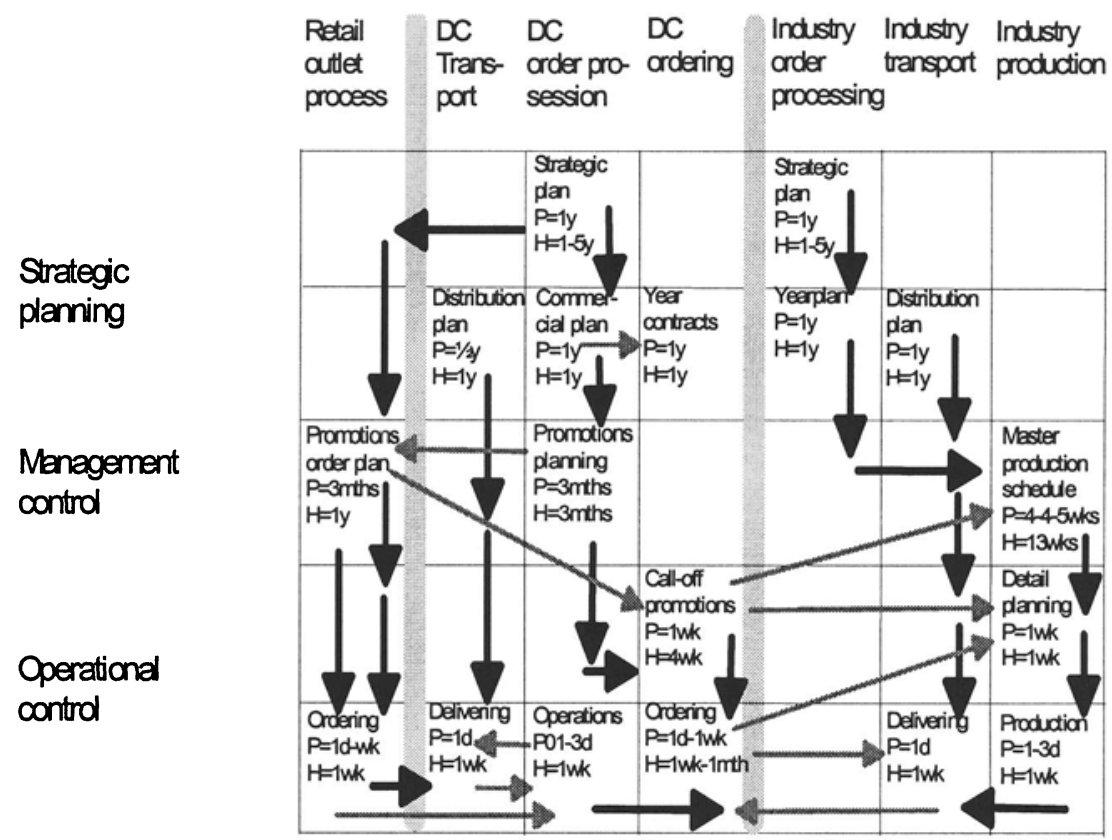

Figure 2 depicts a GRAI Grid for a retail supply chain. In the case we recognise three decision levels: strategic planning, management control, and operational control. Every link in the chain has its own adaptation to these generic decision levels. The processes (horizontal axis) are, for the sake of clarity of the model, aggregates of the processes in the process model in case 1 in this paper. Another simplification of the original GRAI Grid is that external information flows that do not origin in one of the decision centres are left out the model. In the grid decision levels at the lowest level include scheduling activities and execution: e.g. ordering is done once a day to once a week at the retail outlets and takes into account a one-week sales horizon. 
Examples of major opportunities in the chain decision structure:

- long term distribution schedules of the food industry and the retailer should be fine-tuned.

- production plans at the food industry should be fine-tuned with promotion planning at the retailer.

- interval and horizon of detail planning and short term planning (schedules) must be fine-tuned throughout the supply chain to achieve more frequent (e.g., every day) and at the same time smoother deliveries in the supply chain .

\subsection{Activity Chain Model}

The purpose of the Activity Chain Model is "to form activities and chains of activities (business processes) which comply with the increased influence that customers exert on the development and adjustment of products, processes and operations" (Hvolby \& Barfod, 1998). Customer focus and integration are placed as a means to increase effectiveness in conjunction with strategic information systems development as a means to gain competitive advantages. The Activity Chain Model correlates the tasks between the organisation activities and the information systems in the order process within the company. Activity, in this context, means a group of coherent tasks. The purpose of the activity Chain Model is to specify and to place the main activities that are part of the order process by observing the order processing across the activities. The aim of this work is to manage and to control the order process. Meaning, to clarify time consumption, bottleneck problems, transfer of responsibility and costs in connection with administration and production. The activity chain model includes four chains in the basic model: the product development chain, the order chain, the stock chain, and the shop floor chain. In a supply chain context the order chain is an interesting part of the model, as it includes the activities which have to be balanced through out the supply chain.

In the case the business processes in the example chain will be analysed. This was also done in the EPC chart, but focus is a bit different in the ACM chart. Each chain illustrates a coherent decision sequence and the main concern is: forward flow, few changes of responsibility and a minimum use of resources. In this case we have set up a chain for each level in the supply chain: the outlet, the DC and the food manufacturer. Furthermore, the physical goods flow is the food manufacturer (this could be expanded to illustrate the flow to the outlet). The Activity Chain Model is illustrated in figure 3 


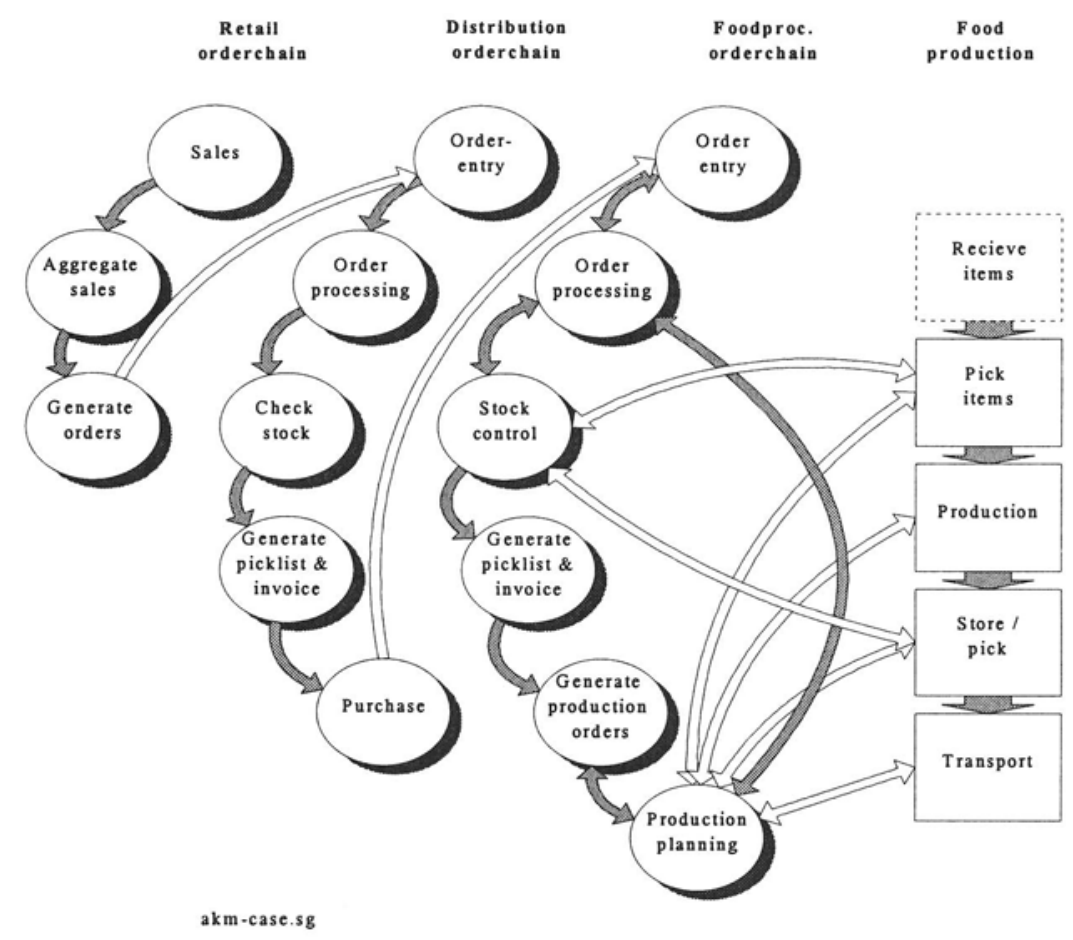

Figure 3.

The order chains are exceptionally straight which are caused by the fact that the processes already have been improved using the EPC model. In reality a great number of feedbacks is observed in the order flow.

\section{CONCLUSION}

Major advantages of the EPC modelling approach are its focus on lead time performance of processes, which makes it quite simple to use. Major advantages of $\mathrm{ACM}$ are the integral approach of concurrent process chains. It aims at efficient use of resources. ACM distinguish between physical tasks in the production and order processing tasks in the administration. The reason for this is that a growing part of the resources in companies are used for administrative tasks. In its analysis it looks critically at the non-value adding activities. EPC does not distinguish between physical tasks in the production and order processing tasks in the administration. This is due to the fact that the main point of interest in this model is lead time reduction, involving both production and administrative tasks. Compared to ACM the EPC model add's the time-dimension and draws attension to delays whereas the relations to other tasks are missing. 
GRAI aims at improvement of decision structures. In designing a decision structure much attention must (naturally) be paid to information flows; these form the major enabler of integration of any kind. In this way GRAI Grids fit with respect to the lower decision levels well with EPC models, in which redesigned information flows also play a major role. Also it fits well with AMC on the lower and medium level decisions, as far as ACM reconfigures and optimises information flows between processes and decisions involved. However when only the order chain is included the value of ACM in the supply chain is limited to the transitional integration between the links in the supply chain.

In chain analysis an important characteristic of chains must be beared in mind. A link in a chain always has multiple relationships with suppliers and customers. This means that bilateral integration of throughput times, decisions and use of resource and tasks always implies a simplification of reality. However we think that the models presented in this paper offer a good communication means to start redesign processes. The case-study shows that the models complement each other, and based on the experiences the following procedure has come into existence:

- identify and correlate the value-adding tasks using the Activity Chain Model.

- identify and reduce lead times using the Event Process Chain.

- identify and correlate decision structures using GRAI Grids.

Future work will include integration and further elaboration and teasing of methods for supply chain analysis and redesign. An important element of this will be the comparison of model application in various chains. The aim is to eventually come to an integrated toolkit of models for chain analysis and redesign.

\section{REFERENCES}

Coll F., A.S.Carrie, U.S.Bititci, A.Reid, J.H.Trienekens and H.H.Hvolby (1998) The implications of interrelationships for decision making in companies along the supply chain. in: Proc. Int. Conf. of the Manufacturing Value Chain. University of Strathclyde, Glasgow. Kluwer Academic Publishers.

Davenport, Thomas H (1993) "Process Innovation", Harward Business School Press.

Doumeingts, G. (1985) How to decentralise decisions through GRAI models in production management. Computers in Industry, No. 6 North Holland Publishing.

Hvolby, Hans-Henrik; Barfod, Ari (1998) "Modelling Customer Order Processes". In: Proceedings of the 13'th IPS Research Seminar on Design for Integration in Manufacturing, Fuglsoe, Denmark

Kim Y.G. Process modeling for BPR (1995) Event-process chain approach. In: Proc.16th Int.Conf.on Information Systems. Eds. J.I.DeGross et al. ICIS. New York.

Lalonde, B.J. \& Pohlen T.L. (1996) Issues in supply chain costing. International Journal of Logistics Management, Vol. 7, No. 1. 
Lee, H.L., V. Padmanabhan V. and S. Whang (1997) The bullwhip effect in supply chains. Sloan Management Review, Vol. 38, Spring.

Porter M. E. (1985) Competitive Advantage, The Free Press.

Van der Vorst, J.G.A.J., A.J.M. Beulens, W. de Wit, P. van Beek, (1998) Supply

Chain Management in Food chains: improving performance by reducing uncertainty, International Transactions in Operations Research, Vol. 5, No. 6, 487-499

Ziggers G.W. and J. Trienekens (1999) Quality Assurance in Food and Agribusiness Supply Chains: Developing successful Partnerships. in: International Journal of Production Economics, 60-61, 271-279. 